\title{
ESTUDIO DE IMPACTO AMBIENTAL EN UNA PLANTA DE DESGASIFICACIÓN Y CUBICACIÓN DE TANQUES CISTERNA PARA EL TRANSPORTE DE COMBUSTIBLES
}

Elmer Tupia *

\author{
RESUMEN
}

El presente Estudio de Impacto Ambiental (EIA) es realizado por la Empresa Ecoplanet Group del Perú S.A. a solicitud de Servisya S.A., propietario de una planta de desgasificación y cubicación de camiones cisterna para transporte de combustibles líquidos derivados de los hidrocarburos, estando el ámbito de la zona de emplazamiento comprendido en la Provincia de Lima del distrito de Villa El Salvador.

Palabras claves : Impacto ambiental. Regulación. Monitoreo.

\section{ABSTRACT}

The present Study of Environmental Impact (EIA) it is carried out by the Company Ecoplanet Group from the Peru CORP. to application of Servisya CORP., proprietor of a desgasificación plant and cubicación of trucks cistern for transports of derived liquid fuels of the hydrocarbons, the environment of the location area understood in the County of Lima of the district of Villa El Salvador being.

\section{INTRODUCCIÓN}

El presente Estudio de Impacto Ambiental (EIA) fué realizado a solicitud de la Empresa Ecoplanet Group del Perú S.A. para una planta de desgasificación y cubicación de camiones cisterna para transportes de combustibles líquidos derivados de los hidrocarburos, estando el ámbito de la zona de emplazamiento comprendido en el distrito de Villa El Salvador, provincia de Lima.

La planta está ubicada a $25.50 \mathrm{~km}$. al sur de Lima y su localización exacta está en la antigua Carretera Panamericana Sur Km. 25.50 Conchan del distrito de Villa EI Salvador de la provincia y departamento de Lima, lugar con afluencia de tráfico y por donde pasan camiones cisterna para la carga y transporte ligero y pesado de combustibles líquidos desde la planta Conchan a los distintos puntos de requerimientos ubicados tanto en la ciudad de Lima, como en las ciudades y/o lugares diversos del interior del Perú.

El objetivo principal de este Estudio es el de evaluar las condiciones durante las actividades tanto de instalación y operación de la planta como, de abandono de ésta si así fuera el caso; considerando evaluaciones en las actividades propias de la planta y en los servicios complementarios, tanto del medio

*Ingeniero Industrial. Instituto de Investigación. Facultad de Ingenieria Industrial. UNMSM.

E-mail : d260040@unmsm.edu.pe emisor como del receptor, que permita determinar el potencial de receptividad ecológica de la zona, en base a mediciones e información existente.

El EIA evalúa la relación del proyecto y el medio ambiente físico que se verá afectado positiva o negativamente en su ecosistema e indicará las medidas de previsión y control que deben ser aplicadas para el desarrollo armónico entre las operaciones de la planta y el ecosistema.

El Estudio se ha dividido en tres etapas, las que interrelacionadas entre sí, permitirán alcanzar la conclusión definitiva de la evaluación de los impactos positivos y negativos para la planta de desgasificación y cubicación. Estas etapas son:

a. análisis preliminar de la información existente,

b. reconocimiento del campo, del medio físico y biológico; y

c. trabajo final de compendio y conclusión en gabinete.

\section{DESCRIPCIÓN DE LA PLANTA DE DESGASIFICACIÓN Y CUBICACIÓN}

La planta de desgasificación y cubicación ocupa un terreno que abarca un área de $2,004.75 \mathrm{~m}^{2}$ con las siguientes medidas perimétricas: por el frente (lado oeste), colinda con la antigua carretera Panamericana Sur, con $36.45 \mathrm{~m}$., por el costado derecho (lado sur), colinda con una propiedad de terceros "Multiservicios Arsi S.R.Ltda.", con 55.00 m., por el costado izquierdo (lado norte), colinda con propie- 
dad de terceros "Sr. Abraham Vizcardo Valdivieso", con $55.00 \mathrm{~m}$. y por el fondo (lado este), colinda con otra propiedad de terceros "Sr. Albino Ponce Yaguno y Sra. Olinda Pamela Ponce Yalico", con 36.45 m.

El muro perimetral que colinda por el lado frontal y laterales derecho e izquierdo tiene una altura de $4.50 \mathrm{~m}$. y el muro perimetral que colinda por el fondo tiene una altura de $4.00 \mathrm{~m}$. ya que ésta se ubica a $2.00 \mathrm{~m}$. desde del nivel del patio de maniobras, haciendo un total de $6.00 \mathrm{~m}$. con respecto al nivel terminado del patio de maniobras.

La distribución de la zona administrativa es la siguiente: Oficinas, servicios higiénicos de la administración, caseta de control, servicios higiénicos para el público, servicios higiénicos más vestuario para el personal operativo, duchas, sala para personal de seguridad y vigilancia.

La disiribibución de las áreas dentro de la planta son: área de desgasificación, área de cubicación, área para colocación y soldadura de la flecha de tanques, área de bombas y ablandadores y área para el tanque de agua. El área no ocupada se considera como área de maniobras de las unidades que requerirán servicios dentro de la planta.

Se considera un radio de giro de $14.0 \mathrm{~m}$. tanto para la entrada y salida como para cada una de las plataformas con que cuenta la planta de desgasificación y cubicación, medidos desde el centro de eje de la entrada y salida. El ángulo tanto de la entrada como de la salida con respecto a los limites del borde interior de la calzada serán de $45^{\circ}$.

En la zona no existen veredas ni bermas señaladas por la Municipalidad distrital de Villa El Salvador ni por e Ministerio de Transporte y Comunicaciones, sin embargo, el proyecto considera reservar las distancias de $2.00 \mathrm{~m}$. para la vereda y $2.00 \mathrm{~m}$. para la berma colindante, que separarán las entradas de la antigua carretera Panamericana Sur con una altura de vereda de $0.20 \mathrm{~m}$. con respecto al nivel de losa terminada del patio de maniobras con una carpeta asfáltica de 2" de espesor y una pendiente del $3 \%$ en los tramos de unión con respecto a la carretera.

La prestación de los servicios de cubicación se realizará a través de plataformas independientes. Se considera tres plataformas ubicadas una al ingreso en forma perpendicular a la Carretera Panamericana Sur y las otras dos en paralelo a la misma antigua carretera Panamericana Sur.

La zona para atención de los camiones cisternas comprende tres estaciones para la prestación de los servicios, siendo estos :

- Primera estación de servicio:Desgasificación

- Segunda estación de servicio:Cubicación
- Tercera estación de servicio : Colocación y soldadura de flecha.

Se ha considerado dos plataformas para la prestación de los servicios de cubicación con 3 puntos de atención, una con atención por ambos lados de la plataforma ; éstas están ubicados uno en línea continua a la puerta de entrada y la otra plataforma (de doble atención) formando un ángulo de $90^{\circ}$ con respecto a las vías de entrada y salida, es decir, paralelo a la Carretera Panamericana Sur.

Las plataformas disponen de escalera de acero marca greenline de 30 " de ancho y barandas de protección con un brazo de carga por la parte superior tipo pantográfico marca EMCO WHEATON con tubería de $f$ 4" $y$ un medidor de flujo electromagnético marca ISOIL con sensor para brida de $f 4$ ".

Además la planta dispondrá de los siguientes equipos :

- Un ablandador de agua de operación semiautomática por medio de una válvula multiport, marca acquamatic de 1", modelo WS2 de 61 m3 de volumen de agua de regeneración, con un flujo máximo de $25 \mathrm{gpm}$ y una presión de operación de 35 psi de ingreso y 25 psi de salida con 36 toberas

- Un sistema de vapor con colector de f $200 \mathrm{mmx}$ $450 \mathrm{~mm}$ de longitud con aislamiento, con $64 \mathrm{~m}$ de tuberías de vapor $\mathrm{SCH}-40$ y un purgador automático de final de línea, una trampa tipo balde invertido con descarga hacia el tanque recibidor de agua de camiones.

- Un sistema de bombeo para tanque de almacenamiento de agua blanda para un caudal de 25 a $30 \mathrm{gpm}$ con una bomba de hidrostal de $1.8 \mathrm{H}$.P y 25 m de tubería de 11 ".

- Un sistema de bombeo para agua de trasiego con una bomba auxiliar hidrostal B1 de 3.4 H.Py $40 \mathrm{~m}$. de tubería de $\mathrm{f} 1$ ".

- Un sistema de llenado de agua hacia los camiones con una bomba hidrostal de 8.6 H.P y $35 \mathrm{~m}$ de tubería de 4 "

La Planta de desgasificación y cubicación cuenta con las siguientes medidas de seguridad: dos extintores de tipo $A B C$ de $12 \mathrm{~kg}$. de capacidad de Polvo Químico Seco, cilindros con arena, letreros pintados en los muros de ambos lados de la propiedad como: "No fumar", "Prohibido hacer fuego abierto a menos de 50 m.", "Prohibido el estacionamiento diurno y nocturno de vehículos", "Prohibido el comercio ambulatorio a menos de $20 \mathrm{~m}$.".

\section{DESCRIPCIÓN DEL MEDIO AMBIENTE}

El área se ubica en el flanco oeste de la cordillera occidental de los Andes en la cuenca del valle del río Lurin, en la llanura aluvial formada por la Cuenca del río Lurin y su desembocadura en el Océano Pacífico, a corta distancia de las estribaciones costeras formadas por el Cerro Lomo de Corvina y 
las riberas del Océano Pacífico, al sur de la ciudad de Lima en el distrito de Villa El Salvador, zona circundante a la planta de Conchan de propiedad de Petróleos del Perú S.A.. La zona se encuentra en el km. 25.50 de la carretera Panamericana Sur y se caracteriza por ser de tipo rústico y semi industrial liviana.

En función de la clasificación del riesgo sísmico de la ciudad de Lima y alrededores, la planta se encuentra en un nivel de riesgo medio o regular, sin embargo se contempla la posibilidad de ocurrencia de sismos de grado 8 en la escala de Mercali.

En los alrededores colindantes a la planta no existen edificaciones, a excepción de los de la planta Conchan (a una distancia de entre 100 y $200 \mathrm{~m}$.) y, los pocos que existen (que se encuentran semi-terminados), que están construidos a partir de ladrillos $y$ adobes $\sin$ tarrajear, se ubican en los lados laterales de la planta. Se aprecian edificaciones de un piso. Por la zona frontal planta (cruzando la carretera y para el lado de la playa Conchán) se aprecia la constitución de terrenos destinados para centros de esparcimiento y para el lado posterior se aprecia el inicio del Cerro Lomo de Corvina que colinda con la población de Villa El Salvador de baja condición social. No se aprecian edificios, ni existen locales comerciales de propiedad particular como restaurantes, panaderías, farmacias, librerias, fotoestudios; tampoco existen áreas destinadas a parques municipales.

En las pocas zonas agrícolas cercanas a la planta, mayormente de consumo doméstico, se aprecia poca vegetación, con presencia de vegetación silvestre y árboles diversos como cipreses y eucaliptos que en su gran mayoría no son propias de ésta zona.

La zona transitable colindante a la planta es la carretera Panamericana Sur, que se encuentra completamente asfaltada, estando el área aún pendiente de urbanizar, por lo que no dispone de los servicios básicos como agua y desagüe; en cambio, si dispone de servicios como luz y teléfono.

\section{Entorno fisico}

La estructura del suelo en la zona de estudio se encuentra conformada tanto por materiales provenientes de los desplazamientos y depósitos originados tanto por las aguas del río Lurin y del Océano Pacífico como los provenientes por los desplazamientos gravitacionales del arenal de la zona colindante y del Cerro Lomo.

El área de estudio se encuentra asentada en una terraza aluvial de relieve a desnivel, que en general, presenta una pendiente que varía entre $3 \%$ a $6 \%$. Los microrelieves existentes propios de la acción aluvial de la cual son derivados, han sido modificados favorablemente por la actividad del hombre, nivelándola ligeramente para propósitos de constitución de industria liviana, tratando de abarcar en zonas cercanas para la construcción de viviendas ligeras (la extensión del área urbana llega a 1500 m. de la planta

Las características climáticas del área de estudio es el que corresponde a desierto pacífico subtropical de temperatura media anual de $20^{\circ} \mathrm{C}$, precipitaciones pluviales escasas constituidas por lloviznas con un promedio de $25 \mathrm{~mm}$. al año, particularmente en otoño e invierno.

La planta producirá una proporción mínima de contaminantes gaseosos que sean dispersados por los vientos de orientación sur-oeste en la zona que recorren a una velocidad entre $7.00 \mathrm{~m} / \mathrm{s}$ y $8.00 \mathrm{~m} / \mathrm{s}$.

El agua a utilizar en la planta de desgasificación y cubicación es proveniente del pozo perforado en la parte lateral del terreno. El nivel se la napa freática se ubica entre $5.00 \mathrm{~m}$. y $7.00 \mathrm{~m}$. de profundidad, del cual se traslada mediante una electrobomba para su almacenamiento en dos tanques cisternas de concreto, derivándolo posteriormente por gravedad, a las líneas sanitarias de la edificación y a los servicios de atención para la cubicación dentro del patio de maniobras.

\section{Entorno biológico}

En las zonas circundantes se observa la presencia de pocos animales, entre los que se puede mencionar mayormente los perros, que es animal doméstico. Igualmente se observa la presencia de insectos como zancudos y mosquitos. Entre las aves más comunes se encuentran los pájaros y palomas, además se observa la presencia de aves marinas que se presentan por temporadas en la zona costera (ubicada a 4.00 y $5.00 \mathrm{~km}$. para el lado de la playa).

La poca vegetación y flora nativa predominante está caracterizada por la presencia de especies ecológicamente adaptadas a la zona entre los que se encuentran los árboles como eucaliptos y cipreses entre los más preponderantes.

Estas especies se ubican en zonas que forman sus propios ecosistemas y que albergan una fauna $y$ flora que no se encuentran con prohibiciones de crianza o en peligros de extinción y que no se ubican en zonas de biodiversidad reservada por no requerirlo

\section{Entorno socio-económico-cultural}

Los asentamientos humanos mas cercanos se ubican a $1500 \mathrm{~m}$. al norte de la planta de desgasificación y cubicación. Las pocas viviendas colindantes no cuen- 
tan con servicios de agua y desagüe; pero, si disponen de luz eléctrica y teléfonos con ligeras deficiencias, sin la presencia de veredas y/o áreas públicas por ser zona que aún no se encuentra urbanizada.

El nivel cultural de la población circundante a la zona de la Planta de desgasificación y cubicación, puede clasificarse como de condición media y media-baja .

La agricultura no es una actividad a la cual se dedica la población del lugar como medio de subsistencia, debido al uso de carácter comercial que se le está dando a las tierras y a la ampliación, sin descuidar las zonas de ampliación urbana que están evaluando las autoridades del distrito. La baja calidad del suelo para el cultivo se demuestra por la poca presencia de una vegetación y arborización

Es característico ver que la zona frontal de Conchan, corresponde a áreas destinadas para playas de buen uso en épocas de verano, sin embargo no es una zona de vegetación frondosa y que la caracteriza por no disponer de abundantes edificaciones, a pesar que el clima es beneficioso, no sólo para la flora del lugar sino también para sus pocos habitantes..

En la zona directamente involucrada y en un radio aproximado de $10 \mathrm{~km}$. a la redonda no se observa la presencia de restos arqueológicos, y que de existir no serían afectados por la operación de la planta de desgasificación y cubicación.

\section{REQUISITOS DE REGULACIÓN}

Los aspectos legales que enmarcan a la construcción y operación de esta planta de desgasificación y cubicación de tanques cisternas para transporte de combustibles líquidos dentro del sector hidrocarburos del país en relación al medio ambiente, se resumen en los reglamentos, normas, pautas que además regulan la calidad del ambiente, salud y seguridad, protección de áreas sensibles, protección de especies amenazadas, si fuera el caso control de explotación y modificación del suelo, así como de las normas que regulan la construcción o modificación en sí como : el Código del Medio Ambiente y de los Recursos Naturales D.L. N ${ }^{\circ} 613$ del 8 de Setiembre de 1990, Reglamento para la Protección Ambiental en las actividades de Hidrocarburos D.S.N046-93-EM y D.S.09-95-EM, Reglamento de Seguridad para el almacenamiento de Hidrocarburos D.S.N052-93-EM y Reglamento General de Construcción entre otros dispositivos.

\section{IDENTIFICACIÓN Y EVALUACIÓN DE LOS IMPACTOS AMBIENTALES}

Los problemas presentados y/o a presentarse durante las tres etapas mencionadas, de carácter enunciativo mas no limitativo (pudiendo encontrar otros no previstos durante las operaciones de la planta), serían la reducción del uso del área del suelo debido a las dimensiones de la planta, las demoliciones y la remoción de los suelos por apertura de zanjas para la cimentación, construcción de las fosas para los pozos de agua, cisternas, séptico, de percolación y del pozo para el tratamiento de las grasas y barros, las posibles contaminaciones por efluentes líquidos que se generarían en la planta por los efluentes domésticos a partir de los servicios higiénicos.

Los impactos ambientales se presentan en las diferentes etapas del proyecto:

Etapa de construcción: Esta etapa se iniciará con acciones para definir la distribución de los elementos que intervienen en el planeamiento y ejecución de las operaciones en la construcción de la planta, que implicará una serie de actividades que se circunscriben a la remoción de edificaciones existentes, las facilidades para los accesos, zanjas, cimentación, encofrados, preparación de los fierros, excavación de los pozos para el tratamiento de grasas y barros, cisternas, pozo séptico y pozo de absorción y demás zanjas para el tendido de las líneas de desagüe, líneas eléctricas y drenajes, etc.

Etapa de operación: Es la etapa en donde los impactos que ocasiona el proyecto tendría efectos ambientales más variables, los que, de producirse sin ningún control podrían ocasionar impactos ambientales críticos.

El sistema de almacenamiento de agua reciclada se realiza en tanques protegidos con muros de contención en fugas y para que no alcancen a tener contacto con el suelo. Se emplearán equipos nuevos y adecuados para las maniobras de desgasificación y cubicación.

\section{EFECTOS AMBIENTALES}

- En la Salud: Generalmente las causas se originan a partir del sistema de trabajo, siendo estas calificadas como leves, siendo en su mayoría de cantidades mínimas, a excepción de los accidentes que se producirían a pesar del plan de seguridad e higiene industrial que debe disponer la planta.

- En la flora y la fauna; Las causas o efectos que pudieran generarse en el área de influencia es mínima, ya que la planta contará con la protección contra la contaminación ambiental, sobre todo si existe poca vegetación y de fácil reproducción como los eucaliptos. Si bien no existen muchas viviendas cercanas, tampoco existen bosques ni especies silvestres protegidos, puesto que el área no corresponde a zona protegida por no existir flora y fauna en peligro de extinción. 
- En los ecosistemas: La modificación del perfil por las construcciones y operaciones a realizar no modifica la morfología del terreno, debido a ser un área que se encuentra en proceso progresivo de modificación por el hombre por extensión del área industrial de la zona, hecho que no implicaría mayor remoción o retiro de material.

- En los recursos hídricos: Los efectos en el agua que pudiera causar los efluentes provenientes de esta actividad no son negativas porque dichos efluentes serán evacuados directamente a un pozo séptico y luego a un pozo de percolación, después de separarse las grasas en las trampas de grasas y barros. El río que pudiera informarse como mas cercano a la planta de desgasificación y cubicación es el río Lurin, no afectándose por la evacuación de los efluentes debidamente tratados que van a ser absorbidos libres de posibles contaminación dentro de los pozos de percolación que pudieran emplearse para crear e irrigar áreas de cultivo de la zona.

- En el suelo: Los efectos en el suelo son casi nulas, a excepción de la perforación de las fosas para la cisterna, trampa de grasas y barros y pozas, que serán debidamente protegidas mediante estructuras de concreto, para evitar un contacto directo con el suelo para el caso de la trampa de grasas y barros. Igualmente se realizarán perforaciones para el pozo de agua, pozo séptico y pozo de absorción, que también estarán debidamente protegidos tanto para la obtención y conservación del agua limpia de posibles contaminantes como para la evacuación de sólidos y líquidos acumulados como efluentes de la planta de desgasificación y cubicación.

- En el aire: Contaminación y desmejoramiento de la calidad del aire por consecuencias de: desgasificación de combustibles de los camiones cisternas dando lugar a emisiones gaseosas con el consiguiente peligro de incendio $\mathrm{y} / \mathrm{o}$ contaminación del medio ambiente, emanaciones de gases fétidos de los residuos sólidos expuestos al medio ambiente y contaminación excesiva del pozo séptico de desagües por verter aguas grasosas sin considerar la evacuación previa por la trampa de grasas y barros.

- En el medio socioeconómico: Problemas de salud y consiguiente desmejoras de la calidad de vida debido a :molestia al medio ambiente por la polvareda y el ruido, durante las obras en la planta de desgasificación y cubicación, contaminación del suelo por las perforaciones de los pozos, riesgos de incendios por derrames de combustibles y emisiones gaseosas provenientes de los suelos contaminados con combustible.

Los impactos de los otros medios serán moderados y un porcentaje menor la ocurrencia de impactos críticos que en la medida de lo posible deberán evitarse.

\section{PLAN DE MANEJO AMBIENTAL}

El Plan de Manejo Ambiental para la Planta de desgasificación y cubicación debe contener las medidas de protección, control, mitigación y contingencia, a seguirse para prevenir y/o minimizar impactos negativos y maximizar aquellos impactos que son beneficiosos. Se contemplarán las normas técnicas para la protección del medio ambiente referente a: programas de monitoreo de efluentes líquidos y de emisiones al aire, además del plan de abandono.

\section{Medidas de seguridad}

- Los camiones cisternas se estacionaran dentro de las instalaciones sobre la losa de concreto cerca de la zona de cubicación. La losa evitara las filtraciones al suelo en caso de probables fugas durante las operaciones. Siempre se atenderá cisternas con conexiones herméticas en buen estado.

- Colocar bandejas para goteo y sobre todo verificar que el tanque del camión cisterna esté completamente vacía o que las válvulas estén cerradas antes de conectar la manguera de descarga de agua para cubicación.

- El ingreso y salida de los camiones cisternas se realizará en forma ordenada y a bajas velocidades si es posible con la ayuda del personal de la planta de desgasificación y cubicación con el fin de evitar accidentes de tránsito al interior.

- Se verificará el tipo cubículo del tanque al cual se va a cubicar; a fin de evitar derrames por sobrellenado, se verificará el volumen del tanque receptor.

- Se debe realizar un control de inventario mensual a través de la medición del volumen en los cisternas y pozas. Generalmente la medición de las pozas se realiza con una varilla para medir la profundidad con el cual deberá realizar un balance volumétrico y se evalúa si hay o no filtraciones de los tanques cisternas y/o trampa de grasa.

De sospecharse que hay filtraciones inspeccionar a mas detalle o efectuar otras evaluaciones para descartar la presencia de fugas.

\section{Programas de monitoreo}

- De efluentes líquidos: se tomará una muestra compuesta de los residuos con una frecuencia trimestral.

- De emisiones gaseosas: Se efectuará monitoreo semestral

- De suelos: mensualmente verificar que no hay presencia de hidrocarburos residuales de los clientes. 


\section{Plan de contingencia y respuesta de emergencia}

- Objetivos: Proporcionar la información necesaria para la toma de decisiones a fin de afrontar los posibles incendios y/o derrames al interior de la planta, de tal forma que se cause el menor impacto sobre la vida humana, los recursos naturales y la infraestructura de la zona. ols

- Asignación de funciones y responsabilidades: Es obligación de todo el personal de la planta de desgasificación y cubicación, conocer y observar las reglas de prevención de incendio y procedimientos de emergencia contenido en este plan.

- Acciones de respuesta: La persona que detecta el derrame debe informar de inmediato al Supervisor (Jefe en Acción)., El Jefe en Acción dará indicaciones a la Brigada y a su vez notificará al Coordinador del Plan de las operaciones que se esián realizando. De ser necesario, se activa el plan de contingencia, las brigadas procederán a cumplir sus funciones, de acuerdo al plan de contingencia para actuar en casos de que esta se produzca.

- Compensación por pérdidas o daños: Deberá contratar además de la póliza general para sus instalaciones, para el personal operativo, para sus productos, una póliza que cubra la responsabilidad civil extracontractual por daños a terceros por un monto mínimo de 200 U.I.T.

- Decomisionado y abandono: La planta de desgasificación y cubicación tiene un estimado de duración de aproximadamente 30 años, estimación realizada en función a los cambios tecnológicos de equipamiento y transportes y movilidades (clientes) que con el uso del gas podría ya no ser de utilidad este tipo de empresas comerciales. Tiempo que se estima tomando en consideración que los tanques pueden tener este tiempo de duración. Sin embargo, una vez construida la planta de desgasificación y cubicación, se espera prestar los servicios en forma indefinida efectuando el mantenimiento adecuado.

\section{COMPROMISOS DEL PROPONENTE}

Medidas para controlar los contaminantes del aire

El deterioro del aire es mínimo al evacuarse pequeños porcentajes de volátiles de los productos por venteo que no perjudican el aire, teniendo previsto un control permanente de estos.

No habrá contribución de material participado por tener losas de circulación asfaltadas y pequeñas áreas verdes para absorber ciertos contaminantes. La planta considera que los contaminantes provie- nen de la trampa de grasas, para lo cual se dispone que tendrán un sistema de limpieza y ventilación hacia el aire para cuando los gases generados se encuentre relativamente estática, permitiendo dar seguridad al medio ambiente.

El control para evitar la emisión de polvos se da desde el momento que consideran que la zona de transitabilidad de la planta de desgasificación y cubicación, esto es, el patio de maniobras, será debidamente asfaltado $\mathrm{y} / \mathrm{o}$ enlosado.

Se observa por tanto que, el control de los contaminantes del aire se circunscribe a colectar los gases generados y a minimizar la generación de otros gases.

\section{Medidas para controlar el agua}

La principal contaminación del agua es por los efluentes domésticos e industriales. La planta de desgasificación y cubicación no ha considerado evacuar ningún tipo de efluente fuera de la red sanitaria, teniendo en cuenta evacuar a través de una trampa de grasas y barros para retener estos elementos y permitir que el agua vaya dentro de los límites aceptables para ser enviados tanto al pozo séptico y luego al pozo de absorción.

Medidas para controlar los contaminantes del suelo

La planta de desgasificación y cubicación tiene un especial cuidado con los desechos provenientes del patio de maniobras, los mismos que no tendrán incidencia en el suelo en forma directa ya que se enlosará todo el área del patio de maniobras.

Para controlar la contaminación del suelo con partículas provenientes de los desechos sólidos domésticos e industriales, siendo estos de efectos negativos, se dispone de cilindros para la recolección de residuos domésticos para ser entregados al recolector municipal como basura, los que son acumulados en cilindros para su evacuación, debidamente identificados y con guías de remisión y salubridad, evitando así cualquier contaminación en el área.

\section{Medidas para controlar el ruido}

Los impactos de las emisiones sonoras se producen sobre los componentes del medio físico, aunque para prever estos impactos se estima niveles sonoros que pueden producirse en la etapa de construcción y operación. Durante la etapa de construcción el nivel sonoro se considera como puntual y temporal, producida por los golpes de los encofrados, los fierros de columnas y la mezcladora, adicionalmente puede estimarse el nivel sonoro del grupo electrógeno que puede ser empleado, mien- 
tras que en la etapa de operación los niveles sonoros son de carácter continuos y permanentes, producidos en su mayoría por las unidades usuarias.

\section{Medidas para controlar el calor}

Para las áreas de las oficinas se emplea materiales que impiden el paso de energía calórica del sol y de equipo que pudiera emplearse que genere calor. En la planta de desgasificación y cubicación no se emplean equipos que generen calor.

\section{CONCLUSIONES}

Con la finalidad de dar cumplimiento a las normas establecidas para la protección ambiental referente a la actividad de desgasificación y cubicación, el Estudio de Impacto Ambiental realizado a la Planta concluye lo siguiente:

- El total del efluente tendrá un proceso de retención de grasas y barros, cuyo efluente irá a un pozo séptico y luego a un pozo de absorción en condiciones óptimas.

- Las emanaciones generadas por la acción del grupo electrógeno (funcionamiento eventual) son de muy poca consideración y de mínima incidencia en el medio ambiente.

- Las condiciones que presenta el proyecto, referente a las pistas de tránsito operacional y áreas verdes, inciden con un impacto positivo referente a la contaminación del suelo.

- Las condiciones dadas por el proyecto y las Normas de Calificación del Reglamento Nacional de Construcciones garantizan y dan la seguridad al proyecto en forma positiva.

- Las condiciones de operación y seguridad en las operaciones son positivas para el trabajador.

- El efecto en la salud del trabajador es de una mínima por el sistema moderno del proyecto.

- Los efectos que se producirán en la salud del trabajador son de tendencia positiva por la seguridad y sistema de construcción y diseño a desarrollarse en el proyecto.
- El efecto sobre la flora y fauna no tiene incidencia negativa por encontrase el proyecto en una zona agrícola semi-industrial que no se encuentra protegida y no tiene prohibiciones ni registra extinciones de flora ni de fauna. Por otro lado la Planta de desgasificación y cubicación mejorará el aspecto estético de la zona (carretera).

- Los efluentes generados de la zona de engrase de vehículos no tienen acción negativa en el medio ambiente si éstos son tratados técnicamente para que no perjudiquen al pozo séptico. La Planta de desgasificación y cubicación dispone de una trampa para grasas.

- Los residuos sólidos no presentan efecto negativo ya que se encuentra en una zona de recojo municipal de basura.

- Desde un criterio socioeconómico, la planta de desgasificación y cubicación es de carácter positivo por la generación de empleos laborales para los pobladores de la zona.

- El efecto es positivo por considerar la planta de desgasificación y cubicación como una mejora de calidad de vida en la zona.

- La modificación geomorfológica no tendrá efecto negativo en la variación del modelaje del suelo y cambio de estructura.

\section{RECOMENDACIÓN}

Es necesario que la planta de desgasificación y cubicación, implemente, controle, supervise y perfeccione permanentemente los métodos y técnicas a fin de minimizar los impactos.

\section{BIBLIOGRAFÍA}

1. American Society for Testing Materials. ASTM 1980. Vol. 5. USA

2. Harrison, L. 1996. Manual de Auditoría Medioambiental, Higiene y Seguridad. Edit. Mc Graw Hill Interamericana. México.

3. Ministerio de Energía y Minas. 1993. Reglamento de seguridad para el almacenamiento de Hidrocarburos. D.S. -93-EM. 